\title{
Electron Damage of CdSe Quantum Rods
}

\author{
S. Maccagnano ${ }^{*}$, K.A. Mkhoyan ${ }^{*}$, J. Calcines $^{* *}$, T.D. Krauss ${ }^{* *}$, J. Silcox ${ }^{*}$ \\ *School of Applied and Engineering Physics, Cornell University, Ithaca NY 14853 \\ ${ }^{* *}$ Department of Chemistry, University of Rochester, Rochester, NY 14627
}

Semiconductor quantum dots and quantum rods have come under close scrutiny in the past decade because of their potential for use in technological and biological applications $[1,2]$. (S)TEM is valuable in understanding the basic properties of these nanoparticles (NPs). Size and shape can be found by analyzing bright and dark field images [3]. The crystal structure along the length of a rod can be determined with nanodiffraction techniques [4]. The chemical make-up and distribution of material in core/shell quantum dots can be measured for an individual dot and along multiple directions on one dot [5]. All of these techniques, however, expose the NP to a potentially damaging electron beam, so damage mechanisms need to be understood and observational limits on some techniques need to be determined.

This study focuses on the damaging effects of the electron probe on CdSe quantum rods, determining the extent of damage by the contrast change in ADF images at the probe location and by the change of the amount of a particular atom under the probe using EELS analysis. This analysis is done for two different configurations of the rod and substrate in relation to the probe.

Figure 1 shows the two different configurations of the sample with the probe. In Case I the probe (green arrow) strikes the NP (orange circle) first and then encounters the carbon substrate (blue film). In Case II the order is reversed.

Damage to the NP can be detected by use of ADF images. As atoms are ejected from the crystal by the electron beam, the sample is thinned and the intensity of the damaged spot decreases. Figure 2 shows typical results for the two cases mentioned above. Case I shows no apparent intensity change in the probe area (at the center of the image) even after more than three minutes exposure to the focused electron probe. In contrast, Case II shows a remarkable change in intensity at the location of the probe after the same amount of exposure. This decrease in intensity in the ADF image indicates a reduction in material thickness and gives evidence for the ejection of atoms from the crystal.

A more quantitative measurement of damage can be achieved using EELS. The Cd Medge is collected as a function of electron beam exposure time, and the area under the edge is used to determine the relative abundance of $\mathrm{Cd}$ under the probe. Figure 3 shows one such measurement. The black squares show the abundance of Cd for Case I, and no significant reduction of $\mathrm{Cd}$ atoms is detected by EELS even after over three minutes of exposure. The protection afforded crystals by a layer of carbon on the exit surface of the sample has been observed before [6,7]. In Case II, however, the ejection of $\mathrm{Cd}$ atoms from the NP is easily detected as the red dots show in Fig. 3. This ejection takes place even though preliminary calculations of the threshold energy needed to eject $\mathrm{Cd}$ atoms from the surface of $\mathrm{CdSe}$ in the wurtzite structure is higher than the beam energy of the 
electron probe $(100 \mathrm{keV})$. A possible conclusion is that the surface $\mathrm{Cd}$ binding energy is weakened by prior ejection of the lighter Se atoms [7].

[1] A.P. Alivisatos, Nat. Biotechnol. 22, 47 (2004).

[2] M. Kazes, et al., Adv. Mater. 14, 317 (2002).

[3] S. Maccagnano et al., Microsc. and Micro. 11, Suppl. 2 (2005) 1458CD.

[4] Z. Yu, et al., App. Phy. Lett. 86, 013101 (2005).

[5] Z. Yu, et al., Nano Lett. 5, 4 (2005) 565.

[6] N.J. Zaluzec, J.F. Mansfield, Microsc. and Micro. (1986) 708.

[7] D.Muller, J. Silcox, Phil. Mag.A 71 (1995) 1375.

[8] This work is supported by Nanoscale Science and Engineering Initiative of the NSF under Award No. EEC-0117770. Funding for the growth of the quantum rods (U. Rochester) was supported by NSF and DOE. Support for STEM operations comes from the Cornell Materials Research Center supported by NSF through grant \#9632275. Also, thanks to M.Thomas for technical support.
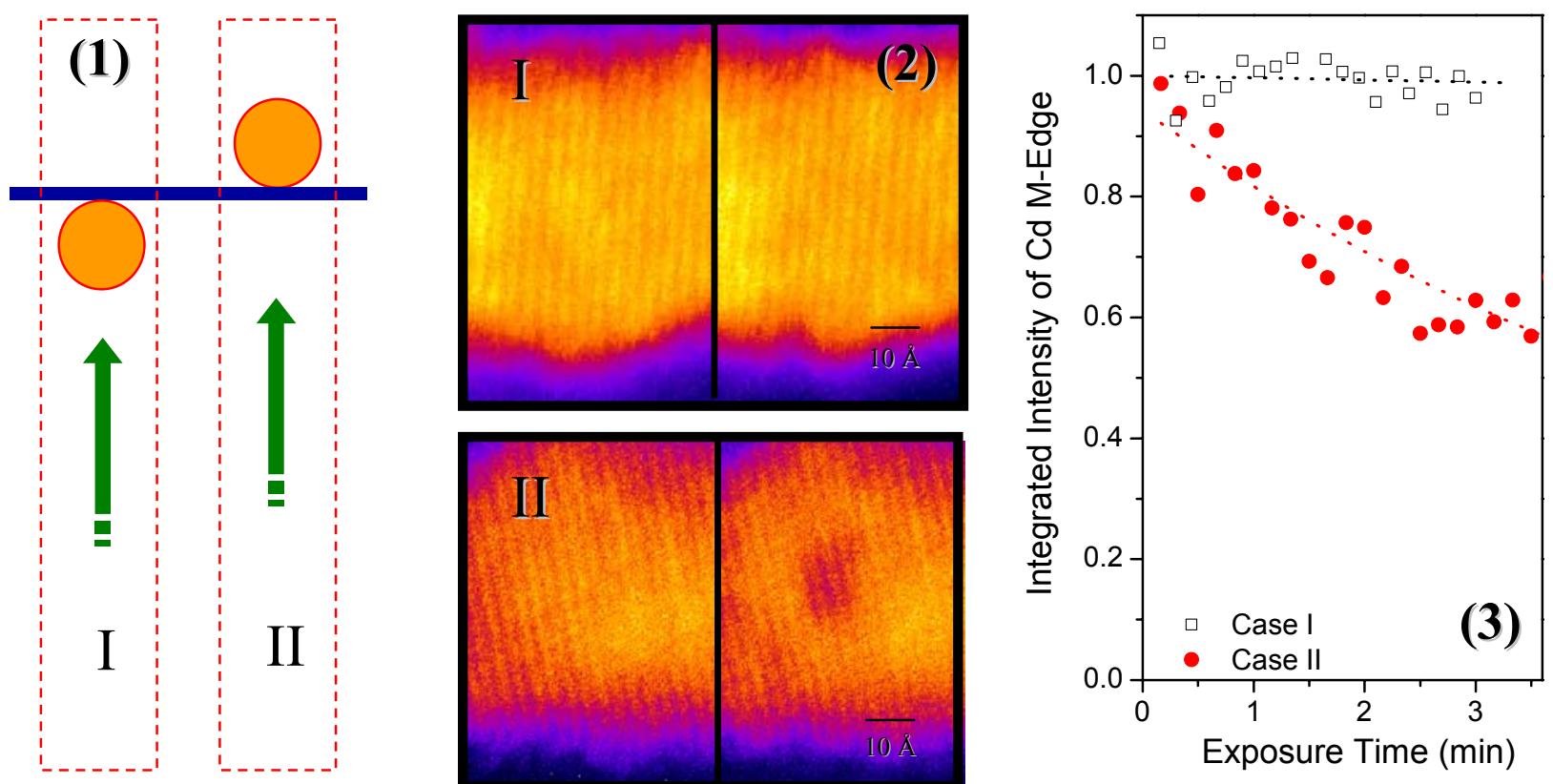

Fig. 1. Different configurations of the QR and carbon substrate. Case I: The electron beam strikes the QR first and then the carbon. Case II: The electron beam strikes the carbon first and then the QR.

Fig. 2. High-resolution ADF images of QR areas before and after electron beam bombardment. Case I shows no obvious change in ADF contrast with beam damage while Case II shows a visible reduction of intensity in the damage area.

Fig. 3. Accompanying EELS data for Case I and Case II in Fig. 2. The area under the Cd M-edge shows no decrease for Case I, but in Case II a marked drop in area under the Cd EELS peak indicates the loss of Cd atoms suffered by the QR. 